\title{
Interfacial and bulk electronic properties of complex oxides and buried interfaces probed by HAXPES
}

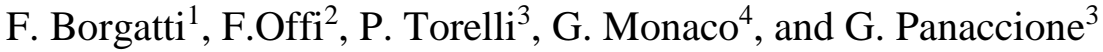 \\ ${ }^{1}$ CNR - Istituto per lo Studio dei Materiali Nanostrutturati (ISMN), Via Piero Gobetti 101, \\ 40129 Bologna, Italy \\ ${ }^{2}$ CNISM and Dipartimento di Fisica, Università Roma Tre, Via della Vasca Navale 84, \\ I-00146 Rome, Italy \\ ${ }^{3}$ CNR, Istituto Officina dei Materiali (IOM), Lab. TASC, in Area Science Park, S.S. 14 - \\ km 163.5, I-34149 Trieste, Italy \\ ${ }^{4}$ European Synchrotron Radiation Facility, BP 220, 38043 Grenoble, France
}

\begin{abstract}
Designing, understanding and controlling the properties of engineered and functional materials, based on oxides and buried interfaces, is one of the most flourishing research fields and one of the major challenges faced by contemporary solid state science and technology. Often, a reliable spectroscopic analysis of such systems is hindered by surface effects, as structural distortion, stoichiometry changes, strong reactivity to external agent and major atomic and/or electronic reconstruction to name but a few. Hard X-Ray PhotoEmission Spectroscopy (HAXPES) is a powerful technique to overcome such limitations, allowing to monitor truly bulk sensitive properties. We report selected HAXPES results for manganese-based oxides, both in films and crystal forms, and for buried metal -organic interfaces, with the aim of highlighting some of the important features such technique brings in the analysis of electronic properties of the solids.
\end{abstract}




\section{Introduction}

Materials science based on surface and interface effects has led to a tremendous theoretical and experimental effort, evolving, in the last four decades, from 'classic' surface science to the advent of 'nanoscience'. Surface and interface properties, however, cannot necessarily be predicted from those observed at larger or smaller scales and are not simply scalable from bulk or atomic properties. In some cases a wealth of new physics has been discovered at these spatial limits of the solid state: interfaces of otherwise well understood solid materials have been shown to exhibit properties much different from those of their bulk parents. Examples range from topologically protected conducting states of systems insulating in the bulk [1] to two dimensional electron gases forming at the interface of two otherwise insulating oxides [2], from unusual magnetic/electric effects, as e.g. the colossal magnetoresistance in magnetic oxides $[3,4]$ to orbitally/magnetically driven effects in buried interfaces [5,6]. Although it is clear that there are many promising new routes to investigate, a significant step forward will be hardly obtained, both for applied and fundamental research, until experimental and theoretical advances will be reached in fully disentangling surface/interface effects and bulk properties. In such systems, and in particular in strongly correlated ones, the marked sensitivity to stoichiometry (layer polarity) or chemical composition (doping) make difficult their experimental and analytical study and often calls for a direct probe of the involved low energy scale phenomena. PhotoEmission Spectroscopy (PES) possesses all the necessary characteristics in elucidating the abovementioned rich physics, but often fails to unravel truly bulk properties and/or cannot access buried interfaces, due to its strong surface sensitivity (probing depth limited to $0.5-2 \mathrm{~nm}$ for typical PES kinetic energy range of 20$1500 \mathrm{eV})$. The use of Synchrotron Radiation has recently boosted Hard X-Ray PhotoEmission Spectroscopy (HAXPES), a technique capable of truly bulk sensitive 
analysis via the measure of high kinetic energy electrons, while keeping energy resolution comparable to low energy PES [7,8]. Moreover, HAXPES experiments not only gave information from a much larger probing depth but also revealed new 'bulk-only' features in transition metal oxides (mainly $3 d$ and $4 d$ ) and $4 f$-based systems, not visible in surface sensitive PES $[9,10]$. In this review an attempt is made to capture some of the original and novel features of the HAXPES technique, with a special focus on $3 d$-based transition metal oxides and buried interfaces.

\section{Experimental}

HAXPES experiments are 'photon hungry'; this is mainly due to the severe reduction of photoionization cross section when increasing the photon energy up to the 5-15 $\mathrm{keV}$ energy range $[11,12,13]$. For this reason, HAXPES spectrometers are usually located at high intensity, undulator based, beamlines. In this paper, all results presented, except the ones in Figure 4, have been obtained at a dedicated setup installed on the ID16 beamline of the European Synchrotron Radiation Facility (ESRF) in Grenoble (France): the VOLPE (Volume PhotoEmission) spectrometer [14]. At ID16, the incident beam is generated by three consecutive undulators and premonochromatized by a liquid-nitrogen cooled doublecrystal $\mathrm{Si}(111)$ monochromator. The bandwidth from the premonochromator is $1.1 \mathrm{eV}$ at $10 \mathrm{keV}$ for a $25 \mu \mathrm{rad}$ vertical divergence of the undulator radiation. Additional reduction of the incident-beam bandwidth can be achieved with a channel cut in a total four-crystal $(+,-,-,+)$ configuration together with the premonochromator. Details of the beamline setup and available spectroscopic tools can be found in Ref. [15]. Typical photon energies used in HAXPES experiments, the corresponding analyzer crystals, possible channel-cut reflections, resulting bandwidths, and fluxes at the sample are reported in Table 1. After the monochromator ensemble, the beam can be focused using an Rh-coated toroidal 
mirror, resulting in a typical focal spot size of about $50 \mu \mathrm{m} \times 130 \mu \mathrm{m}(\mathrm{V} \times \mathrm{H})$. Photoelectrons are collected in normal emission in the horizontal plane, so that the angle between the take-off direction and both the propagation and polarization vectors of the beam is $45^{\circ}$. The best overall (beamline + analyzer) energy resolution we obtained with our setup was $70 \mathrm{meV}$ at $\mathrm{h} v=5934 \mathrm{eV}$ [14]. For the experiments presented we used an analyzer setup such to have an overall energy resolution of about $300 \mathrm{meV} / 450 \mathrm{meV}$ in the photon energy range from $6 \mathrm{keV}$ to $8 \mathrm{keV}$, as a good compromise to distinguish the desired spectral features with good statistic in a reasonable time.

\section{Results and discussion}

\section{I-Metal-to-insulator transition in Manganites}

The manganites comprise a large class of strongly-correlated magnetic materials that exhibit the colossal magnetoresistance effect [16], as well as in some cases half-metallic ferromagnetism [17]. They have various possible future applications in spintronics, particularly via their half-metallicity, rendering them as the ideal materials to develop novel concepts of oxide-electronic devices. However, in spite of a great deal of research on their properties, much is still not understood about the complex interplay of atomic structure, composition, electronic charge and spin/orbital ordering. A correct description of their electronic properties via PES is however limited by the different behavior of the surface with respect to the bulk. This is for example evident for the layered compound $\mathrm{La}_{2-2 x} \mathrm{Sr}_{1+2 x} \mathrm{Mn}_{2} \mathrm{O}_{7}$ whose crystal structure is derived from the perovskite structure by interleaving double blocks of rocksalt structure, $(\mathrm{La}, \mathrm{Sr}) \mathrm{O}$, between two perovskite sheets [18], with a period of the resulting layered structure of approximately $1 \mathrm{~nm}$. Such class of manganites shows a quasi-two-dimensional electronic behavior [19] and a strong difference between the outermost $\mathrm{Mn}-\mathrm{O}$ bilayer and the rest on the material has been 
experimentally verified [20]. Moreover, below the ferromagnetic transition temperature $\left(T_{\mathrm{C}}\right)$ the surface would still have an insulating behaviour while the bulk of the system should behave as a metallic-ferromagnet. It is then evident how difficult is the spectroscopic identification via ordinary (e.g. low kinetic energy-low probing depth) PES experiment of the metal-insulator transition in such a system: only the first bilayer would be probed due to the limited information depth. Finally, detailed angular resolved PES experiments with photo-excitation in the vacuum ultraviolet range, revealed the presence of quasi-particles, signature of a metallic state, in the spectral function at selected directions only in momentum space [21, 22], observations later reconciled by the claim of stacking-fault intergrowth of larger number of stacked $\mathrm{MnO}_{2}$ planes [23]. On the other hand, HAXPES experiments offer an information depth up to $20 \mathrm{~nm}$ in normal emission geometry with kinetic energies around $6 \mathrm{keV}$ [24], being able to probe the bulk electronic structure on these bilayer manganites. We followed this approach to identify the change of the electronic properties in $\mathrm{La}_{1.2} \mathrm{Sr}_{1.8} \mathrm{Mn}_{2} \mathrm{O}_{7}$ single crystals upon crossing their $T_{\mathrm{C}}$, nominally at $120 \mathrm{~K}$ [25]. A spectral investigation of both Mn core levels and valence band was performed above and below the magnetic transition temperature [26]; in Fig. 1(a) the O $1 s$ spectrum is reported at temperatures above $T_{C}$ (thick line) and below $T_{C}$ (open symbols connected by thin line), respectively $285 \mathrm{~K}$ and $60 \mathrm{~K}$. One observes that the spectrum taken at $T=60 \mathrm{~K}$ is shifted (by approximately $100 \mathrm{meV}$ ) to lower binding energy compared to the spectrum at higher temperature, and it is more asymmetric, with a higher intensity tail toward higher binding energy. These results can be interpreted as fingerprints of the metal-insulator transition, given the similarity with the results obtained on La1${ }_{x} \mathrm{Sr}_{\mathrm{x}} \mathrm{MnO}_{3}$ thin films [27]. In Ref. [27] the system is brought from metal to insulator at a given temperature by varying the doping level $x$, with a rigid peak shift with hole doping and an asymmetric line shape in the metallic phase. To strengthen such analogy between 
our observation and the results of Ref. [27], we measured the binding energy position of the $\mathrm{O} 1 \mathrm{~s}$ peak at different temperatures: results are reported in Fig. 1(b) as solid symbols with error bars. One observes that the experimental data are grouped around two binding energy values corresponding to two distinct intervals of temperature. While further experiments are necessary to corroborate this conclusion, we attribute this behavior to the crossing of the Curie temperature, in analogy with the binding energy displacement of the O $1 s$ observed in cubic manganite [28]. The effect is here much sharper than what observed for cubic manganite, probably because of the sharp transition of both magnetic and transport measurement of layered manganite as a function of temperature at $\mathrm{T}_{\mathrm{C}}$ as observed by Mitchell et al. [29]. According to the data of Fig. 1(b), a transition temperature of about $105 \mathrm{~K}$ could be deduced, a result compatible with the expected value of $\mathrm{T}_{\mathrm{C}}$ when including a $15 \%$ error of the thermocouple reading the sample temperature.

\section{II-Well-screened satellites in transition metal oxides: manganites films and crystals}

Besides being an excellent spectroscopic tool to identify the metal-insulator transition, HAXPES revealed unique features in strongly correlated systems, almost hidden at lowest, surface sensitive, excitation energy and often connected with the presence of a non-local mechanism of electron screening [30,31]. Among these, particularly important are the additional low-binding-energy features of core level lines, the so-called well screened satellites, firstly observed in Mn $2 p_{3 / 2}$ core level spectra on manganite epitaxial thin films [32]. In this case the extra satellites have been connected with both the metallic and ferromagnetic properties of the systems; in particular, extra peaks disappear both when crossing the Curie temperature $T_{C}$ and when the system enters in the insulating phase $[33,34]$. While the origin of these satellites is not yet completely settled down, theories refer to the presence of screening mechanism that are more important in the bulk 
than in the surface. In particular, according to one model [32], doping-induced states would develop at the Fermi level, serving as the additional screening channels and producing the observed well screened final states. The intensity of the corresponding peaks is related to the hybridization between the additional states and the $3 d$ band, increasing for increased electron localization (more metallicity). On the other hand, nonlocal screening effects from neighboring sites are considered as the origin the additional low-bonding-energy satellites by van Veenendaal's theory [30]. Also within this approach, the relative peaks intensity is related to the electronic and magnetic properties of the sample, for example resulting in a higher intensity for an increased hopping probability at low temperature as a benchmark of an increased magnetic ordering. In previous publications we were able to show the presence of extra satellites not only associated with the $2 p$ lines but also with core levels of other symmetry, as $3 p$ and $3 \mathrm{~s}$; such observation has been ascribed to different overlaps of the core level with valence screening charge [34,35]. A key question regarding the presence or absence of the well screened satellites refers to the possibility of using such structures as the spectroscopic 'signatures' of a metallic-like behaviour in systems close to a metal-insulator transition, and/or the use of such signature for quick and reliable 'quality-control' of samples produced to device applications. In this case, although the samples would be produced in a less controlled experimental conditions (both from the morphological and crystalline point of view), the bulk sensitivity guaranteed by HAXPES may turn into an easier method of control. We have investigated this possibility via HAXPES experiments on $\mathrm{La}_{0.7} \mathrm{Sr}_{0.3} \mathrm{MnO}_{3}$ (LSMO) films of thickness $25 \mathrm{~nm}$ deposited on $\mathrm{SrTiO}_{3}(001)$ substrates by channel spark ablation (CSA) as detailed in Refs. [36,37,38]. Mn $2 p$ core level spectra from the films are compared in Fig. 2 with equivalent spectra obtained from a $\mathrm{La}_{0.7} \mathrm{Sr}_{0.3} \mathrm{MnO}_{3}$ single crystal sample measured at similar temperature (below the Curie 
temperature) and with similar photon excitation energy. In both cases, the extra satellites are clearly visible in the lower binding energy side of the Mn $2 p_{3 / 2}$ peak. Such feature is thus inherent to the electronic structure of this class of sample, independent on the preparation condition and the actual crystallographic perfection or surface termination and can be identified as a general benchmark of the magnetic and metallic status of these classes of materials.

\section{III-Buried interfaces: Co-Alq3}

The HAXPES technique has the necessary kinetic energy resolution and depth sensitivity to probe ex situ the electronic structure of the bulk, hence is a unique tool for the electron spectroscopy of buried interfaces of technological as well of fundamental interest $[39,40]$. This is an advantage especially to study as-grown systems, or in the case that step-by-step deposition methods cannot be employed. As an example, we report from Ref. [41] the chemical characterization of deeply buried organic-inorganic interfaces belonging to hybrid spintronic devices [37,42]. The possible occurrence of perturbing effects such as intermixing, chemical reactions, formation of dipole layers or molecular disruption demands for the study of prototypical systems prepared with the same protocols as for the operational devices. In advance, this study demonstrates the feasibility of HAXPES to probe buried low-Z elements for which the core-level photoionization cross sections in the hard $\mathrm{x}$-ray range are weaker by a factor $10^{-2}$ to $10^{-3}$ with respect to traditional photon energy ranges $[11,12,13]$. Fig. 3 shows HAXPES data from a cobalt ferromagnetic film (top electrode, $15 \mathrm{~nm}$ of thickness) deposited on a thick tris8hydroxyquinoline(aluminum) layer $\left[\mathrm{Al}\left(\mathrm{C}_{9} \mathrm{H}_{6} \mathrm{NO}\right)_{3}\right.$, hereafter named $\left.\mathrm{Alq}_{3}\right]$, with and without a thin interfacial $\mathrm{AlO}_{\mathrm{x}}$ tunnel barrier $(2 \mathrm{~nm})$, and from a reference thick film (50 $\mathrm{nm})$ of bare $\mathrm{Alq}_{3}$. 
In this condition the Inelastic Electron Mean Free Path (IMFP) across the Co layer was about $6 \mathrm{~nm}$ [24], thus providing a depth sensitivity through the Co electrode and into the $\mathrm{Co} / \mathrm{Alq}_{3}$ interface region for about $3 \lambda_{\mathrm{IMFP}}=18 \mathrm{~nm}$ from the surface. The HAXPES measurements probed the $\mathrm{C} 1 s, \mathrm{~N} 1 s, \mathrm{O} 1 s, \mathrm{Al} 1 s$ signals at room temperature. A detailed presentation and discussion of all the spectra can be found in Ref. [41]. Here we summarize the most significant results achieved from the core level spectra for nitrogen and carbon, reported in Fig. 3. Being unaffected by any photoelectron emission from the $\mathrm{AlO}_{\mathrm{x}}$ layer, the $\mathrm{N} 1 s$ and $\mathrm{C} 1 s$ HAXPES spectra, compared to those of a thick bare $\mathrm{Alq}_{3}$ film $(50 \mathrm{~nm})$, provide unambiguous information on the $\mathrm{Alq}_{3}$ electronic structure at the boundary region with cobalt. In particular, the $\mathrm{N} 1 s$ core level spectrum is strongly sensitive to charge transfer to the molecule because the lowest unoccupied molecular orbital (LUMO) is mostly present on the pyridyl $\left(\mathrm{C}_{5} \mathrm{H}_{4} \mathrm{~N}\right)$ than on the phenoxide $\left(\mathrm{C}_{6} \mathrm{H}_{5} \mathrm{O}\right)$ side of the $\mathrm{Alq}_{3}$ ligands. [43, 44]. Besides the shake-up features well known from the literature [45], the $\mathrm{N} 1 s$ spectrum for the $\mathrm{Co} / \mathrm{Alq}_{3}$ interface exhibits a sizable enlargement at the low binding energy side, while the spectrum for the system with the $\mathrm{AlO}_{\mathrm{x}}$ interface layer is basically identical to the one of the bare $\mathrm{Alq}_{3}$ sample. The $\mathrm{C} 1 s$ spectra behave similarly, yet with a smaller broadening. The fit analysis identified these additional spectral features as produced by the increase, mainly localized at the $\mathrm{N}$ atoms, in the local electronic charge of the $\mathrm{Alq}_{3}$ molecules. The HAXPES depth sensitivity into the $\mathrm{Alq}_{3}$ layer is basically limited to $1-2 \mathrm{~nm}$ of the interface, thus the quantitative comparison of the feature intensities is mostly representative of that region. In this case, the intensity ratio within the $\mathrm{N} 1 s$ spectrum yields approximately 1:2 for the reacted:unreacted component, which is consistent with only one of the three nitrogen atoms per molecule being modified by charge transfer [46]. The $\mathrm{O} 1 \mathrm{~s}$ and $\mathrm{Al} 1 \mathrm{~s}$ spectra, not shown here, resemble the same behaviour of the $\mathrm{N} 1 \mathrm{~s}$ and $\mathrm{C} 1 \mathrm{~s}$ spectra without any specific feature or binding energy shift 
that could indicate a different kind of perturbation of the $\mathrm{Alq}_{3}$ electronic structure, e.g. due to molecular fragmentation [46]. In particular, the HAXPES study of the Co/Alq3 interface is in good agreement with a similar experiment performed in situ by XPS and UPS [47]. These results indicate, in the absence of the $\mathrm{AlO}_{\mathrm{x}}$ barrier, the formation of a mixed $\mathrm{Co}-\mathrm{Alq}_{3}$ interface in which cobalt atoms or clusters diffuse within the $\mathrm{Alq}_{3}$ layer and create $\mathrm{Alq}_{3}$ anions by donating electronic charge to each molecule. Therefore the $\mathrm{AlO}_{\mathrm{x}}$ barrier not only reduces significantly the Co-Alq 3 interdiffusion, but also prevents very efficiently any alterations of the $\mathrm{Alq}_{3}$ electronic structure at the interface.

\section{IV- Valence band HAXPES}

A paradigmatic example of the capabilities of HAXPES is the analysis of the valence band states where genuine bulk sensitivity is required. Paying the price of the already mentioned reduction of intensity due to cross section decrease, the experimental density of states (DOS) extracted from valence band spectra collected with kinetic energies up 7-8 $\mathrm{keV}$ can be reliably compared to those generated by a variety of calculation methods, and provides important information about the relationship and difference between bulk and surface properties, as shown by several groups for complex and highly correlated oxides [7,8]. HAXPES valence band experiments on complex oxides are particularly useful for clarifying the role of oxygen, on the one hand by reducing the uncertainties due to surface preparation and/or enrichment or depletion at the surface layers, and on the other hand by obtaining reliable comparison of the DOS in device-ready systems. In the specific case of the manganites, the amount and the preferential location of oxygen vacancies influences e.g. the Curie temperature and the overall resistivity. Conversely, the oxygen deficiencies of manganite thin films can also be employed for tuning the performance of associated devices [48]. Fig. 4(a) displays the HAXPES spectrum of the DOS for a LSMO film of 30 
nm deposited on $\mathrm{SrTiO}_{3}(001)$ substrate by channel spark ablation [38]. In order to gain a deeper insight into the effects of oxygen vacancies on the electronic structure, the spectrum is compared to DFT theoretical calculations (panel b) performed in Ref. [49]. The theoretical curves A-D reproduce the total DOS calculated for defective LSMO, with vacancies placed in different planes of the lattice model, namely A corresponds to $\mathrm{SrO}$ planes, $\mathrm{B}$ and $\mathrm{D}$ to $\mathrm{MnO}$ planes surrounded respectively by $\mathrm{SrO} / \mathrm{LaO}$ and $\mathrm{LaO} / \mathrm{LaO}$ planes, $\mathrm{C}$ to $\mathrm{LaO}$ planes: also the total DOS for the ideal condition is reported in panel $\mathrm{b}$ ). In the calculation, the peak $F_{1}$ is assigned to $\mathrm{O} 2 p$ states, while $\mathrm{F}_{2}$ and $\mathrm{F}_{3}$ are associated to the $\mathrm{Mn} t_{2 g}$ states, and $\mathrm{F}_{4}$ to the $\mathrm{Mn} e_{g}$ states. The presence of oxygen vacancies in the calculations induces the shift and modulation of the density of states with respect to the ideal case, in particular for the $F_{2}$ and $F_{3}$ structures. The experimental data show large similarity with the theory and good agreement for the binding energy position of the $\mathrm{F}_{1}-\mathrm{F}_{4}$ spectral features. The intensities of these terms resemble qualitatively the theoretical curve A, hence suggesting that the bulk of this LSMO thin film includes oxygen vacancies being preferentially located in the lattice planes including the $\mathrm{Sr}$ ions. The Feature $\mathrm{H}$ at around 7 $\mathrm{eV}$ cannot be recognized in the calculations, probably because in the comparison with the XPS results of Ref. [49] they were convoluted with a quite broad Gaussian function accounting for the experimental resolution. However, on the basis of UPS results shown in the same work, this feature has been associated to the shift towards larger binding energy of the oxygen-derived states occurring at about $5 \mathrm{eV}$ due to the presence of oxygen vacancies. The clear occurrence of this feature in the HAXPES spectra, favoured by the larger decay of the cross section for the Mn 3d levels with respect to the $\mathrm{O} 2 \mathrm{p}$ states [11], confirms that in the probed sample the vacancies are largely distributed in the bulk.

The strong variation of cross section when passing from soft to hard x-ray reveals to be an advantage if one would like to highlight the metal $s$ contribution of the total density of 
states in valence band photoemission. In fact, although the cross section of all states is severely suppressed in the HAXPES regime (up to a factor $10^{-4}$ ) [11-13], the relative ratio of states with different symmetry strongly favors extended $s$-states with respect to localized $d$ and $f$ ones, enabling the observation of a well-defined occupied conduction band and a metallic Fermi edge, if present $[8,50,51]$. This is particularly useful for the class of organic semiconductor materials largely involved in the development of hybrid organic/inorganic devices, such as organic field-effect transistors (OFETs), organic lightemitting diodes (OLEDs) [52,53] and, more recently, organic spin valves (OrgSVs) or magnetic tunnel junctions (OMTJs) [36,37]. The knowledge of the electronic properties of these molecular assemblies is essential for the design and performance of any devices derived from these systems, being the nature, size and dynamics of the spin/charge carriers strongly related to the energy distribution of the molecular orbitals and the energy gap of the occupied/unoccupied states, as well as to the amount of intermolecular interaction occurring in the solid state phase. Among the large variety of $\pi$-conjugated molecules exhibiting semiconducting behavior, tris8-hydroxyquinoline(aluminum) $\left[\mathrm{Al}\left(\mathrm{C}_{9} \mathrm{H}_{6} \mathrm{NO}\right)_{3}\right.$, $\mathrm{Alq}_{3}$ ] has been widely used, and largely characterized by conventional ultraviolet/x-ray photoelectron spectroscopy, therefore being a well known prototypical example for the application of HAXPES to organic materials. Fig. 5 shows the HAXPES spectrum of the valence states for a thick $\mathrm{Alq}_{3}$ layer in comparison to conventional spectra acquired in the UV/soft X-ray photon energy range as reported in Ref. [54]. The sample was a thick $\mathrm{Alq}_{3}$ layer of $50 \mathrm{~nm}$, deposited ex situ on a native silicon oxide substrate by thermal evaporation. During the measurements, the sample temperature was set at $100 \mathrm{~K}$ to reduce beam damage effects. We notice that the radiation damage promoted by exposure to the incident x-ray beam may affect not only the organic compounds but, although in lower extent, can occur also for inorganic materials. The electronic defects generated through the 
de-excitation mechanisms following the absorption of the photons can destabilize the compounds to such a degree that recombination is no longer possible. As a result, desorption into vacuum can occur and the compound stoichiometry is changed (see e.g. Ref. [55]). Concerning the hard x-ray regime, this effect cannot be excluded a priori. For example, small but noticeable changes of core level HAXPES spectra have been observed for $\beta-\mathrm{PbO}_{2}$ [56]. However the large absorption length of the hard $\mathrm{x}$-rays within the material and the huge reduction of the photo-excitation cross section should reduce the rate of damage in the probed volume over reasonable time scales, hence leaving the HAXPES spectra unaffected long enough to get reasonable statistics. Conversely, even for the hard x-ray energy range the organic compounds require to translate frequently the sample under the beam to preserve from excessive damage, as it has been done to acquire the DOS spectrum shown in Fig. 5 for the $\mathrm{Alq}_{3}$ thick film. The comparison of the HAXPES data with conventional UPS/XPS spectra exhibits large similarities leading to the clear identification of the spectral features corresponding to the occupied molecular orbitals, hence ensuring about the good quality of the material despite no preliminary treatment to remove surface contaminants has been adopted. The main differences arise for the spectral terms with binding energy lower than $6 \mathrm{eV}$, i.e. the peaks closer to the Fermi level, showing a marked intensity reduction with respect to the ones collected with photon energy $240 \mathrm{eV}$. The theoretical calculation of the projected density of states performed in Ref. [54] indicate that in this binding energy range the spectral features are dominated by the contributions of the carbon and oxygen ions. However the photoionization cross section for oxygen is expected to decay faster than carbon while increasing the photon energy, hence reducing its spectral weight. A rough estimation on the basis of atomic calculations $[11,12]$ indicates that, taking into account the number of $\mathrm{C}$ and $\mathrm{O}$ ions in the molecule, the ratio of the $\mathrm{C} 2 p / \mathrm{O} 2 p$ cross sections raises up from $\approx 1$ to 
$\approx 2.4$ passing from photon energy $300 \mathrm{eV}$ to $8000 \mathrm{eV}$, respectively. Therefore the detection of the valence states by HAXPES appears as a promising tool to distinguish more in detail the spectral features of the low-Z elements in the bulk density of states of organic compounds.

\section{Conclusions}

HAXPES has reached a mature state as a valuable and versatile spectroscopic technique; present applications of the technique cover a broad range of topics from condensed matter physics into materials science, energy science, microelectronics, and nanoengineering to name but a few. In the next future, thanks to the rapid technical developments in detectors efficiency and the increased number of dedicated experimental setup at high intensity/high resolution synchrotron beamlines, HAXPES will open new opportunities in the study of complex systems and buried interfaces. Moreover, the long pathlengths for photoelectrons excited at high energy will enable study of technologically important samples without the elaborate in situ preparation procedures usually necessary in surface science experiments. We anticipate that when the optimized control of external parameters such as electric field, temperature and pressure will be reached, HAXPES activity will play a pivotal role in the, so far unexplored, 'in operando' regime, both at large-scale synchrotron facilities and in laboratory-based environments.

\section{Acknowledgements}

We are very grateful to the colleagues of the VOLPE group and of ID16 beamline at ESRF, and in particular to G. Cautero and P. Lacovig (Elettra), A. Fondacaro, C. Henriquet, R. Verbeni, S. Huotari, L. Simonell, G. Vanko (ESRF). Thanks are due to Jun Fujii for the experimental support and for many fruitful discussions. Thanks to P. Graziosi for the fabrication of the manganite thin films. We would also like to thank W. Drube, S. Thiess and G. Gloskovskii for assistance at the PETRA-III beamline. This work has been supported in part by CNR-INFM. The research leading 
to these results has received funding from the FP72007-2013 framework programme under grant NMP3-LA-2010-246102. The HAXPES instrument at PETRA III beamline P09 is jointly operated by the University of Würzburg (R. Claessen), the University of Mainz (C. Felser) and DESY. Funding by the Federal Ministry of Education and Research(BMBF) under contracts 05KS7UM1, 05K10UMA, 05KS7WW3, and 05K10WW1 is gratefully acknowledged. 


\section{Table Captions}

Table 1. Photon energies typically used for HAXPES experiments and corresponding channelcut schemes, with photon beam bandwidths (meV) and fluxes in photons/s at the sample position (fluxes are given at a ring current of $200 \mathrm{~mA}$ ).

\section{Figure Captions}

Figure 1. (a) $\mathrm{O} 1 s$ HAXPES spectrum measured at $\mathrm{hv}=5931 \mathrm{eV}$ from $\mathrm{La}_{1.2} \mathrm{Sr}_{1.8} \mathrm{Mn}_{2} \mathrm{O}_{7}$ single crystal above (thick line) and below (open symbols) the transition temperature; (b) binding energy position of the $\mathrm{O} 1 s$ peak versus temperature; the dashed line is a guide to the eyes.

Figure 2.Mn $2 p$ core level spectra from single $\mathrm{La}_{0.7} \mathrm{Sr}_{0.3} \mathrm{MnO}_{3}$ crystal (open symbols) and LSMO film (solid line), measured respectively at $\mathrm{h} v=7.7 \mathrm{keV}, 150 \mathrm{~K}$ and $\mathrm{h} v=6.9 \mathrm{keV}$, $\mathrm{T}=200 \mathrm{~K}$, well in the ferromagnetic state. The well screened satellite, indicated by the arrow, is visible in both spectra.

Figure 3. $\mathrm{N} 1 s$ and $\mathrm{C} 1 s$ spectra measured with $\mathrm{h} v=7.6 \mathrm{keV}$ at room temperature for bare $\mathrm{Alq}_{3}$ (red solid line), $\mathrm{Co}(15 \mathrm{~nm}) / \mathrm{Alq}_{3}$ (black squares) and $\mathrm{Co}(15 \mathrm{~nm}) / \mathrm{AlO}_{\mathrm{x}}(2 \mathrm{~nm}) / \mathrm{Alq}_{3}$ (open circles). The peak intensities have been normalized to compare the lineshapes. The analytical fit for the spectra of the $\mathrm{Co} / \mathrm{Alq}_{3}$ interface present an additional feature (CT) due to charge donation from $\mathrm{Co}$ to the $\mathrm{Alq}_{3}$ molecule, more evident for the $\mathrm{N} 1 s$ (brown) than for the C $1 s$ (dark green) spectra. The blue features, located around $291 \mathrm{eV}$ binding energy for $\mathrm{C} 1 \mathrm{~s}$ and $402.5 \mathrm{eV}$ for $\mathrm{N} 1 s$, respectively, correspond to the shake-up terms involving $\pi \rightarrow \pi^{*}$ transitions, while the bulk Alq 3 core-level terms are shown in red (N $\left.1 s\right)$ and green (C $1 s)$, the latter showing the contribution of the different carbon bonds.

Figure 4. (a) HAXPES valence states spectrum of a LSMO film (30 nm grown on STO), measured with a photon energy of $7 \mathrm{keV}$. At this photon energy the information depth is expected to be around $20 \mathrm{~nm}$; sample temperature was $200 \mathrm{~K}$ (i.e. well below the critical temperature of about $\mathrm{Tc} \approx 340 \mathrm{~K}$ ). The positions of the main spectral features are indicated by lines and letters. Good agreement is found with theoretical DFT calculations (b) reported from Ref. [49] of the DOS including the presence of oxygen vacancies at different lattice sites (details in the text and in Ref. [49]). In the inset of panel (b), the crystal structure of LSMO model adopted for the theoretical analysis.

Figure 5. (a) $\mathrm{Alq}_{3} \mathrm{UV} /$ soft $\mathrm{x}$-ray photoelectron spectra compared to theoretical DFT calculations. Figure reported from Ref. [54] (b) HAXPES spectrum of the Alq 3 valence states measured with photon energy $7.6 \mathrm{keV}$. Inset: $\mathrm{Alq}_{3}$ molecular structure. The spectrum was measured at the P09 beamline of the PETRA-III synchrotron light source with a photon energy of $7.6 \mathrm{keV}$; the grazing incidence angle of the $\mathrm{x}$-ray beam was 5 degrees, while the signal was collected at normal emission geometry, with an overall resolution of $0.35 \mathrm{eV}$. 


\section{References}

[1] M. Z. Hasan, C. L. Kane Rev. Mod. Phys. 82 (2010) 3045.

[2] J. Mannhart, D. G. Schlom, Science 327 (2010) 1607.

[3] S. A. Wolf, D. D. Awschalom, R. A. Buhrman, J. M. Daughton, S. von Molnar, M. L. Roukes,

A. Y. Chtchelkanova, D. M. Treger, Science 294, (2001) 1488.

[4] Y. Tokura and Y. Tomioka, J. Magn. Magn. Mater. 200 (1999) 1.

[5] J. Fujii, M. Sperl, S. Ueda, K. Kobayashi, Y. Yamashita, M. Kobata, P. Torelli, F. Borgatti, M.

Utz, C. S. Fadley, A. X. Gray, G. Monaco, C. H. Back, G. van der Laan, G. Panaccione, Phys. Rev. Lett. 107 (2011) 187203.

[6] I. Vobornik, U. Manju, J. Fujii, F. Borgatti, P. Torelli, D. Krizmancic, Y. S. Hor, R. J. Cava, G. Panaccione, Nano Lett., 11 (2011) 4079.

[7] C. S. Fadley, J. Electron Spectrosc. Relat. Phenom. 178-179 (2010) 2.

[8] G. Panaccione, K. Kobayashi, Surf. Science, 3-4 (2012) 125.

[9] G. Panaccione, U. Manju, F. Offi, E. Annese, I. Vobornik, P. Torelli, Z. H. Zhu, M. A. Hossain, L. Simonelli, A. Fondacaro, P. Lacovig, A. Guarino, Y. Yoshida, G. A. Sawatzky, A. Damascelli, New J. Phys. 13 (2011) 053059.

[10] L. Moreschini, C. Dallera, J. J. Joyce, J. L. Sarrao, E. D. Bauer, V. Fitsch, S. Bobev, E. Carpene, S. Huotari, G. Vankó, G. Monaco, P. Lacovig, G. Panaccione, A. Fondacaro, G. Paolicelli, P. Torelli, M. Grioni, Phys. Rev. B 75 (2007) 035113.

[11] J. H. Scofield, Lawrence Livermore National Laboratory Report UCRL-51326 (1973)

[12] M.B. Trzhaskovskaya, V.K. Nikulin, V.I. Nefedov, V.G. Yarzhemsky, At. Data Nucl. Data Tables 92 (2006) 245; M.B. Trzhaskovskaya, V.I. Nefedov, V.G. Yarzhemsky, At. Data Nucl. Data Tables 82 (2002) 257.

[13] J J Yeh and I. Lindau, At. Data Nucl. Data Tables 32 (1985) 1.

[14] P. Torelli, M. Sacchi, G. Cautero, M. Cautero, B. Krastanov , P. Lacovig, P.Pittana, R. Sergo, , R. Tommasini, A. Fondacaro, , F. Offi, G. Paolicelli, , G. Stefani, M. Grioni, R. Verbeni, G. Monaco, G. Panaccione, Rev. Sci. Instrum. 76 (2005) 023909.

[15] R. Verbeni T. Pylkkanen, S. Huotari, L. Simonelli, G. Vanko, K. Martel, C. Henriquet, G. Monaco, J. Synchrotron Rad. 16 (2009) 469.

[16] S. Jin, T. H. Tiefel, M. McCormack, R. A. Fastnacht, R. Ramesh, L. H. Chen, Science 264 (1994) 413.

[17] J.-H. Park, E. Vescovo, H.-J. Kim, C. Kwon, R. Ramesch, T. Venkatesan, Nature (London) 392 (1998) 794.

[18] J. F. Mitchell, D. N. Argyriou, A. Berger, K. E. Gray, R. Osborn, U. Welp, The Journal of Physical Chemistry B 105 (2001) 10731.

[19] H. M. Rønnow, Ch. Renner, G. Aeppli, T. Kimura, Y. Tokura, Nature (London) 440 (2006) 1025.

[20] J. W. Freeland, K. E. Gray, L. Ozyuzer, P. Berghuis, E. Badica, J. Kavich, H. Zheng, J. F. Mitchell, Nat. Mater. 4 (2005) 62.

[21] N. Mannella, W. L. Yang, X.J. Zhou, H. Zheng, J. F. Mitchell, J. Zaanen, T. P. Devereaux, N. Nagaosa, Z. Hussain, and Z.-X. Shen, Nature 438, 474 (2005)

[22] Z. Sun, Y.-D. Chuang, A. V. Fedorov, J. F. Douglas, D. Reznik, F. Weber, N. Aliouane, D. N. Argyriou, H. Zheng, J. F. Mitchell, T. Kimura, Y. Tokura, A. Revcolevschi, and D. S. Dessau, Phys. Rev. Lett. 97, 056401 (2006)

[23] F. Massee, S. de Jong, Y. Huang, W. K. Siu, I. Santoso, A. Mans, A. T. Boothroyd, D. Prabhakaran, R. Follath, A. Varykhalov, L. Patthey, M. Shi, J. B. Goedkoop and M. S. Golden, Nature Physics 7, 978 (2011) 
[24] M. Sacchi, F. Offi, P. Torelli, A. Fondacaro, C. Spezzani, M. Cautero, G. Cautero, S. Huotari, M. Grioni, R. Delaunay, M. Fabrizioli, G. Vankó, G. Monaco, G. Paolicelli, G. Stefani, G. Panaccione, Phys. Rev. B 71 (2005) 155117.

[25] J. F. Mitchell, D. N. Argyriou, J. D. Jorgensen, D. G. Hinks, C. D. Potter, S. D. Bader, Phys. Rev. B 55 (1997) 63.

[26] F. Offi, P. Torelli, M. Sacchi, P. Lacovig, A. Fondacaro, G. Paolicelli, S. Huotari, G. Monaco, C. S. Fadley, J.F. Mitchell, G. Stefani, G. Panaccione, Phys. Rev. B 75 (2007) 014422.

[27] K. Horiba, M. Taguchi, N. Kamamura, K. Yamamoto, A. Chainani, Y. Takata, E. Ikenaga, H. Namatame, M. Taniguchi, M. Awaji, A. Takeuchi, D. Miwa, Y. Nishino, K. Tamasaku, T. Ishikawa, H. Kumigashira, M. Oshima, M. Lippmaa, M. Kawasaki, H. Koinuma, K. Kobayashi, and S. Shin, J. Electron Spectrosc. Relat. Phenom. 144-147 (2005) 557.

[28] N. Mannella, A. Rosenhahn, C. H. Booth, S. Marchesini, B. S. Mun, S.-H. Yang, K. Ibrahim, Y. Tomioka, and C. S. Fadley, Phys. Rev. Lett. 92, 166401 (2004).

[29] J. F. Mitchell, D. N. Argyriou, J. D. Jorgensen, D. G. Hinks, C. D. Potter, and S. D. Bader, Phys. Rev. B 55, 63 (1997).

[30] M. van Veenendaal, Phys. Rev. B 74 (2006) 085118.

[31] G. Panaccione, F. Offi, P. Torelli, and M. Sacchi, Comptes Rendus Physique 9 (2008) 524.

[32] K. Horiba, M. Taguchi, A. Chainani, Y. Takata, E. Ikenaga, D. Miwa, Y. Nishino, K. Tamasaku, M. Awaji, A. Takeuchi, M. Yabashi, H. Namatame, M. Taniguchi, H. Kumigashira, M. Oshima, M. Lippmaa, M. Kawasaki, H. Koinuma, K. Kobayashi, T. Ishikawa, and S. Shin, Phys. Rev. Lett. 93 (2004) 236401.

[33] H. Tanaka, Y. Takata, K. Horiba, M. Taguchi, A. Chainani, S. Shin, D. Miwa, K. Tamasaku, Y. Nishino, T. Ishikawa, E. Ikenaga, M. Awaji, A. Takeuchi, T. Kawai, and K. Kobayashi, Phys. Rev. B 73 (2006) 094403.

[34] G. Panaccione, M. Altarelli, A. Fondacaro, A. Georges, S. Huotari, P. Lacovig, A. Lichtenstein, P. Metcalf, G. Monaco, F. Offi, L.Paolasini, A. Poteryaev, M. Sacchi, O. Tjernberg, Phys. Rev. Lett. 97 (2006) 116401.

[35] F. Offi, N. Mannella, T. Pardini, G. Panaccione, A. Fondacaro, P. Torelli, M. W. West, J. F. Mitchell, C. S. Fadley, Phys. Rev. B 77 (2008) 174422.

[36] V. A. Dediu, L. E. Hueso, I. Bergenti, and C. Taliani, Nat. Mater. 8 (2009) 707.

[37] C. Barraud, P. Seneor, R. Mattana, S. Fusil, K. Bouzehouane, C. Deranlot, P. Graziosi, L. Hueso, I. Bergenti, V. Dediu, F. Petroff, and A. Fert, Nat. Phys. 6 (2010) 615.

[38] V. Dediu, J. López, F. C. Matacotta, P. Nozar, G. Ruani, R. Zamboni, and C. Taliani, Phys. Status Solidi B 215 (1999) 625.

[39] E. Holmström, W. Olovsson, I.A. Abrikosov, A.M.N. Niklasson, and B. Johansson, M. Gorgoi, O. Karis, S. Svensson, F. Schafers, W. Braun, G. Ohrwall, G. Andersson, M. Marcellini, and W. Eberhardt, Phys. Rev. Lett. 97 (2006) 266106.

[40] C. Dallera, F. Fracassi, L. Braicovich, G. Scarel, C.Wiemer, M. Fanciulli, G. Pavia, B.C.C. Cowie, Appl. Phys. Lett. 89 (2006) 183521.

[41] F. Borgatti, I. Bergenti, F. Bona, V. Dediu, A. Fondacaro, S. Huotari, G. Monaco, D. A. MacLaren, J. N. Chapman, and G. Panaccione, Appl. Phys. Lett. 96 (2010) 043306.

[42] V. Dediu, L. E. Hueso, I. Bergenti, A. Riminucci, F. Borgatti, P. Graziosi, C. Newby, F. Casoli, M. P. De Jong, C. Taliani, Y. Zhan, Phys. Rev. B 78 (2008) 115203.

[43] A. Curioni, W. Andreoni, R. Treusch, F. J. Himpsel, E. Haskal, P. Seider, C. Heske, S. Kakar, T. van Buuren, and L. J. Terminello, Appl. Phys. Lett. 72, 1575 (1998).

[44] T.-W. Pi, C.-H. Liu, and J. Hwang, J. Appl. Phys. 99, 123712 (2006).

[45] T.-W. Pi, T.C.Yu, C.-P. Ouyang, J.-F. Wen, and H.L.Hsu, Phys. Rev. B 71 (2005) 205310.

[46] V.-E. Choong, M.G. Mason, C.W. Tang, Y. Gao, Appl. Phys. Lett. 72 (1998) 2689. 
[47] W. Xu, J. Brauer, G. Szulczewski, M.S. Driver, A.N. Caruso, Appl. Phys. Lett. 94 (2009), 233302

[48] F.X. Hu, J. Gao, J. R. Sun, and B. G. Shen Appl. Phys. Lett. 83 (2003) 1869; W.B. Wu, K. H. Wong, C. L. Mak, C. L. Choy, and Y. H. Zhang J. Appl. Phys. 88 (2000) 2068.

[49] S. Picozzi, C. Ma, Z. Yang, R. Bertacco, M. Cantoni, A. Cattoni, D. Petti, S. Brivio, and F. Ciccacci, Phys. Rev. B 75 (2007) 094418.

[50] H.L. Zhang, D.J. Payne, R. G. Palgrave, V. K. Lazarov,W. Chen,A. T. S. Wee, C. F. McConville, P. D. C. King, T. D. Veal, G. Panaccione, P. Lacovig, and R. G. Egdell, Chem. Mater. 21 (2009) 4353.

[51] A. Walsh, Juarez L. F. Da Silva, Su-Huai Wei, C. Korber, A. Klein, L. F. J. Piper, A. DeMasi, K. E. Smith, G. Panaccione, P. Torelli, D. J. Payne, A. Bourlange, and R. G. Egdell, Phys. Rev. Lett. 100 (2008) 167402.

[52] R.H. Friend, R.W. Gymer, A.B. Holmes, J. H. Burroughes, R.N. Marks, C. Taliani, D.D.C. Bradley, D.A. Dos Santos, J.L. Brédas, M. Lögdlund, W. R. Salaneck, Nature 397, (1999) 121.

[53] E. Arisi, I. Bergenti, V. Dediu, M. A. Loi, M. Muccini, M. Murgia, G. Ruani, C. Taliani, R. Zamboni, J. Appl. Phys. 93, (2003) 7682.

[54] F. Bisti, A. Stroppa, M. Donarelli, S. Picozzi, and L. Ottaviano Phys. Rev. B 84 (2011) 195112

[55] R. G. Copperthwaite, Surf. Interface Anal. 2 (1980), 17

[56] D.J. Payne, G. Paolicelli, F. Offi, G. Panaccione, P. Lacovig, G. Beamson, A. Fondacaro, G. Monaco, G. Vanko, R.G. Egdell, Journal of Electron Spectroscopy and Related Phenomena 169 (2009), 26-34 
Table 1

Photon energies typically used for HAXPES experiments and corresponding channelcut schemes, with photon beam bandwidths $(\mathrm{meV})$ and fluxes in photons/s at the sample position (fluxes are given at a ring current of $200 \mathrm{~mA}$ ).

\begin{tabular}{|c|c|c|c|}
\hline Energy & Chunnel-cut & $\begin{array}{c}\text { Incident beam } \\
\text { bandwidth }\end{array}$ & Intensity \\
\hline$(\mathrm{keV})$ & & $(\mathrm{meV})$ & $\left(\right.$ photons s$\left.^{-1}\right)$ \\
\hline 5.93 & None & 650 & $1.4 \times 10^{13}$ \\
\hline 5.93 & $\mathrm{Si}(333)$ & 52 & $6.2 \times 10^{11}$ \\
\hline 6.46 & $\mathrm{Si}(220)$ & 300 & $2.6 \times 10^{12}$ \\
\hline 7.91 & $\mathrm{Si}(444)$ & 39 & $1.1 \times 10^{12}$ \\
\hline 9.69 & $\mathrm{None}$ & 1200 & $7.0 \times 10^{13}$ \\
\hline 9.69 & $\mathrm{Si}(220)$ & 400 & $1.5 \times 10^{13}$ \\
\hline 9.69 & $\mathrm{Si}(440)$ & 200 & $3.5 \times 10^{12}$ \\
\hline 9.69 & $\mathrm{Si}(444)$ & 140 & $1.5 \times 10^{12}$ \\
\hline 9.69 & $\mathrm{Si}(555)$ & 15 & $3.2 \times 10^{11}$ \\
\hline
\end{tabular}




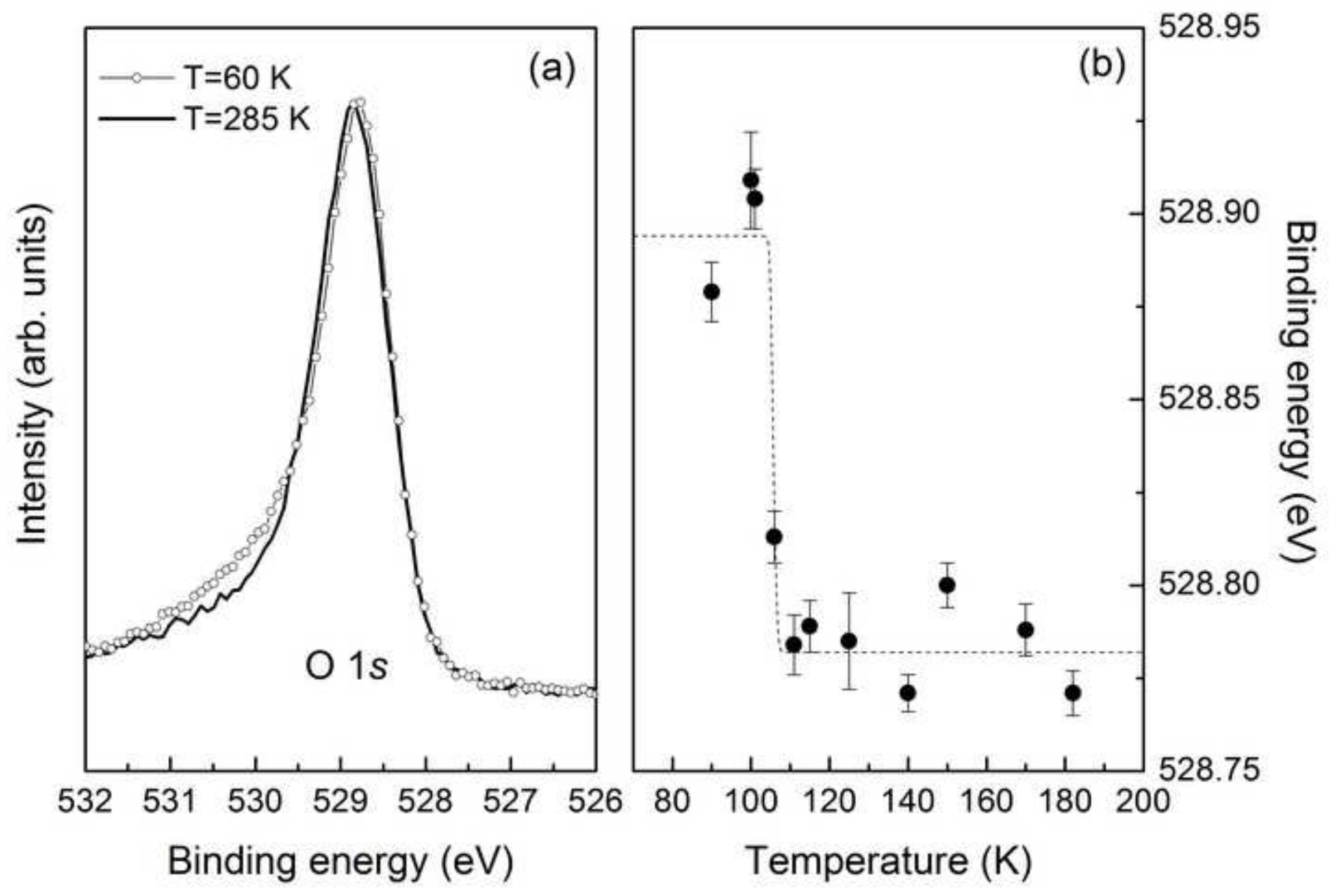




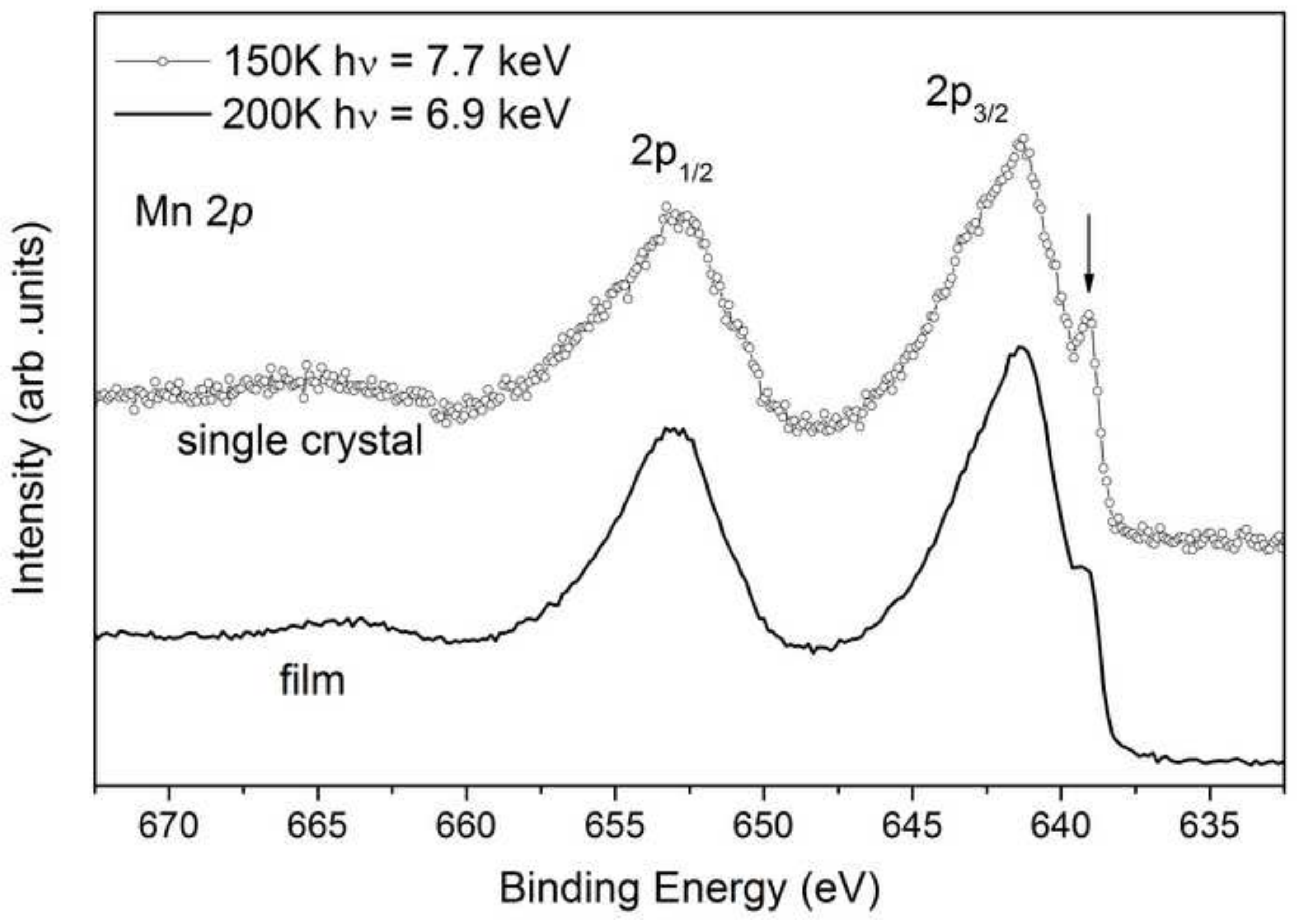




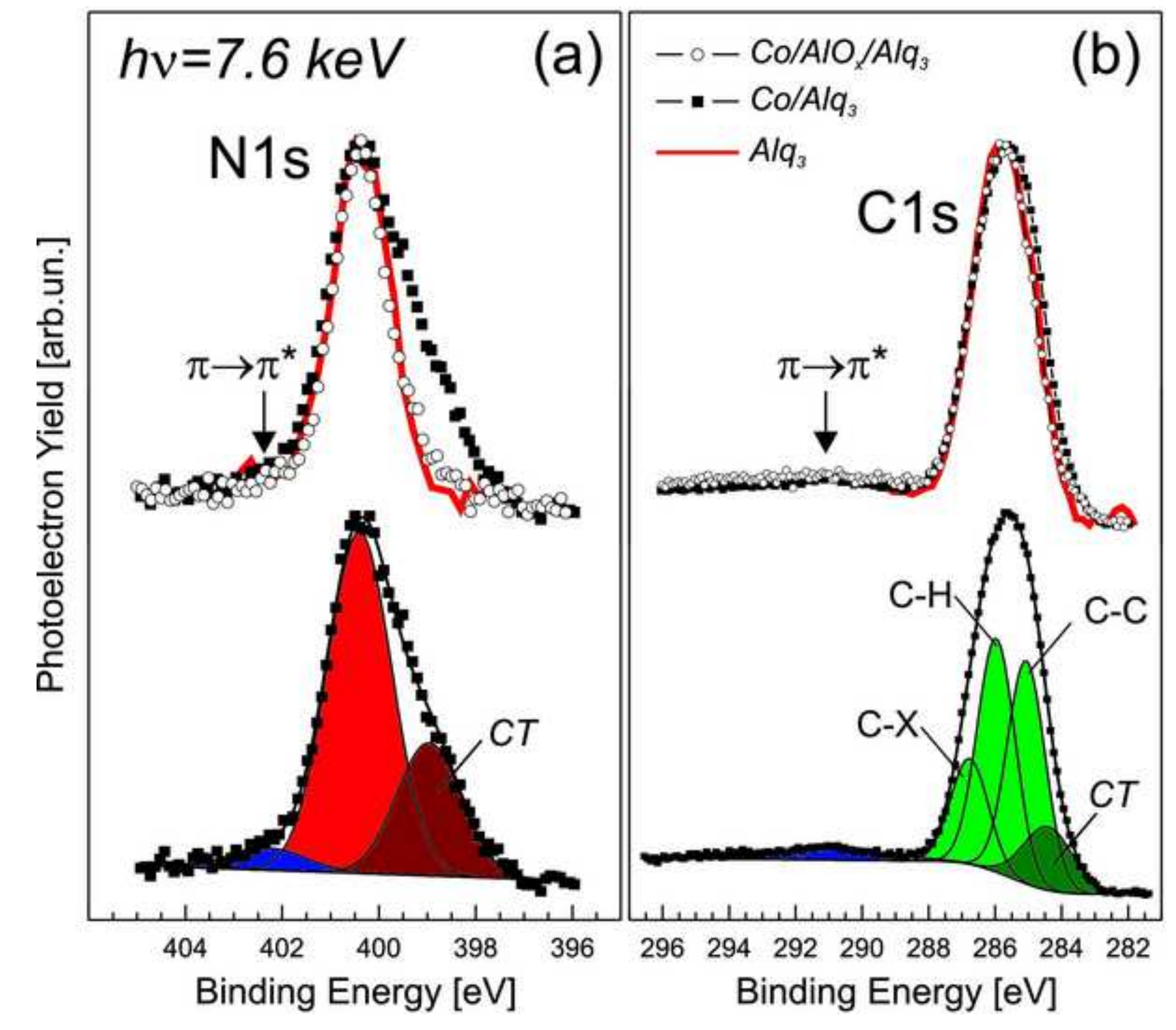



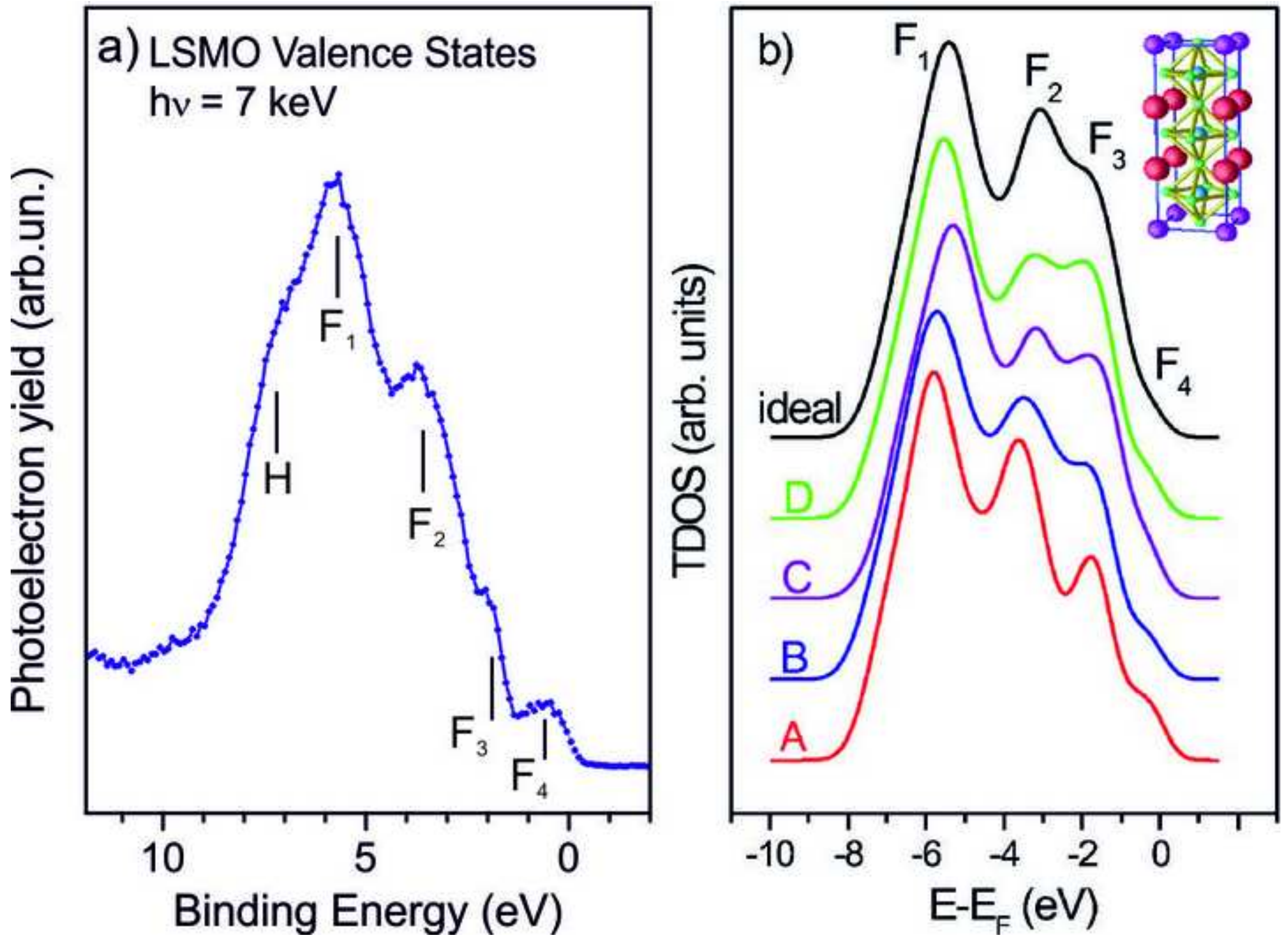


\section{Figure5}

Click here to download high resolution image

Binding Energy (eV)

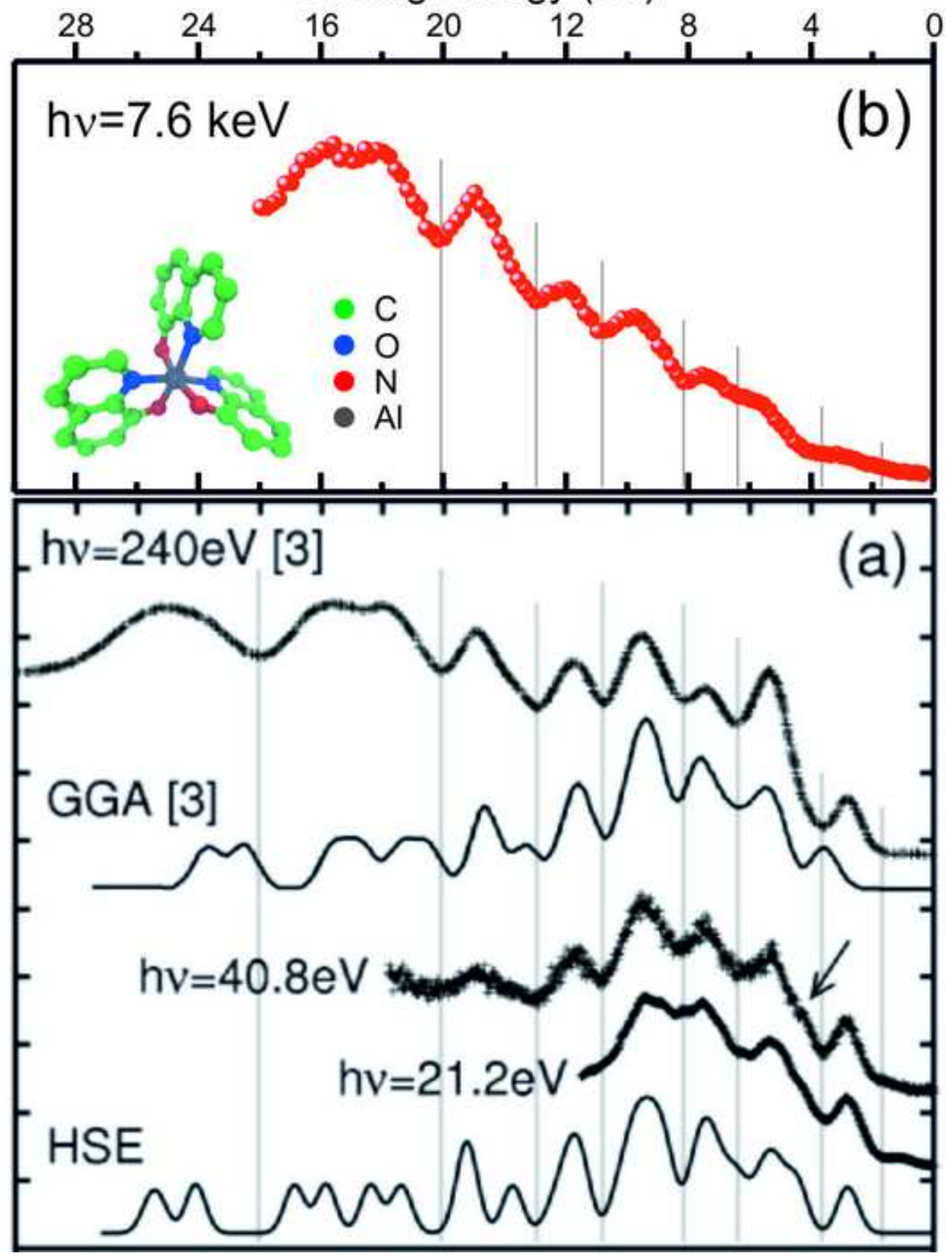

